PROCEEDINGS OF THE

AMERICAN MATHEMATICAL SOCIETY

Volume 132, Number 1, Pages 47-58

S 0002-9939(03)07034-

Article electronically published on May 22, 2003

\title{
CHARACTERIZATION OF CLIFFORD-VALUED HARDY SPACES AND COMPENSATED COMPACTNESS
}

\author{
LIZHONG PENG AND JIMAN ZHAO
}

(Communicated by David R. Larson)

\begin{abstract}
In this paper, the general Clifford $R_{n, s}$-valued Hardy spaces and conjugate Hardy spaces are characterized. In particular, each function in $R_{n}$ valued Hardy space can be determined by half of its function components through Riesz transform, and the explicit determining formulas are given. The products of two functions in the Hardy space give six kinds of compensated quantities, which correspond to six paracommutators, and their boundedness, compactness and Schatten-von Neumann properties are given.
\end{abstract}

\section{INTRODUCTION}

Clifford-valued ( $R_{n}$-valued) Hardy spaces have been the subject of many works, in particular, by F. Sommen, F. Brackx, R. Delanghe, J. Ryan, T. Qian, M. Mitrea, K. Gürlebeck, W. Sprößig, S. Bernstein, etc.; see [10, [11, 3], 9], [5], 44, 1], etc. In

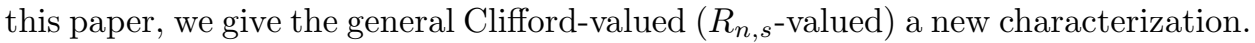

To explain the Hardy space theory on the general Clifford algebras $R_{n, s}$, let us go back to the classical Hardy space theory on the complex numbers $C$. Denote the square-integrable complex-valued function space on the real line $R$ by $L^{2}(R, C)$. The classical Hardy space $H^{2}(R, C)$ is defined to be

$$
H^{2}(R, C)=\left\{f \in L^{2}(R, C) \mid F(x+i y)=P_{y} * f(x), \frac{\partial F}{\partial \bar{z}}=0\right\},
$$

where $P_{y} * f(x)$ is the Poisson integral of $f(x)$. By Fourier theory it turns out to be

$$
H^{2}(R, C)=\left\{f+i H f \mid f \in L^{2}(R, R)\right\}=\left\{f \in L^{2}(R, C) \mid \operatorname{supp} \widehat{f} \subset[0,+\infty)\right\} .
$$

In this space, each complex-valued function $f+i H f$ is determined by its real component. There is a decomposition $L^{2}(R, C)=H^{2}(R, C) \bigoplus \bar{H}^{2}(R, C)$, where $\bar{H}^{2}(R, C)$ is the conjugate Hardy space.

In this paper, we generalize these to the general Clifford algebras $R_{n, s}$ and give the characterizations of the general Clifford-valued Hardy spaces and conjugate Hardy spaces. In particular, $R_{n}$-valued Hardy spaces are characterized, and each

Received by the editors November 15, 2001 and, in revised form, September 4, 2002.

2000 Mathematics Subject Classification. Primary 15A66, 42B30, 46J15, 47B35.

Key words and phrases. Clifford algebra, Cauchy-Riemann operator, Hardy space, compensated compactness, paracommutator.

Research supported by NNSF of China Nos. 90104004 and 69735020 and 973 project of China G1999075105. 
function in this kind of Hardy space is determined by half of its function components through Riesz transform. Moreover, for those representations, the explicit formulas are given. Comparing with the decomposition of square-integrable function space on the real line $R$ into the direct sum of Hardy space and conjugate Hardy space, the square-integrable Clifford algebra-valued function spaces are decomposed into the orthogonal sum of Clifford-valued Hardy and conjugate Hardy spaces.

In the classical case, taking two functions $f=f_{0}+i H f_{0}$ and $g=g_{0}+i H g_{0} \in$ $H^{2}(R, C)$, one has studied the product $f g=f_{0} g_{0}-H f_{0} H g_{0}+i\left(f_{0} H g_{0}+H f_{0} g_{0}\right)$; both its real part and imaginary part have compensated compactness. They are called compensated quantities. A one-to-one correspondence between paracommutator $T_{b}(A) f$ and compensated quantity $Q_{A}(f, g)$ has been established in [8]. The paracommutator was studied systematically by Janson, Peetre and Peng ([6], [7]). For the special case, the corresponding relation is as follows: $\langle f g, b\rangle=\left\langle H_{b} f, \bar{g}\right\rangle$. Therefore the BMO-boundedness, VMO-compactness and Schatten-von Neumann properties of $H_{b} f$ or $f g$ can be read in theorems in [8]. In [13, 14], Zhijian Wu studied the product of a left monogenic function and a right monogenic function. In this paper, we shall consider the product of two left monogenic functions in Hardy spaces. This gives a nature method to obtain some examples of compensated quantities.

In $\S 2$ we give some preliminaries. In $\S 3$ we characterize the Hardy space $H^{(k)}\left(R^{n}, R_{n, s}\right)$. By the characterization of Hardy space, we give some examples of the compensated quantities and paracommutators arising from the product of two functions in Hardy space.

\section{Preliminaries}

This section is an overview of some basic facts which are concerned with Clifford algebras. We set up the general formalism which will be used in the sequel (cf. [2]).

Let $V_{(n, s)}(0 \leq s \leq n)$ be an $n$-dimensional $(n \geq 1)$ real linear space with basis $\left\{e_{1}, \cdots, e_{n}\right\}$, and provided with a bilinear form $(v, w), v, w \in V_{(n . s)}$, such that $\left(e_{i}, e_{j}\right)=0, i \neq j ;\left(e_{i}, e_{i}\right)=1, i=1, \cdots, s ;$ and $\left(e_{i}, e_{i}\right)=-1, i=s+1, \cdots, n$.

If $v=\sum_{i=1}^{n} v_{i} e_{i} \in V_{(n, s)}$, then the associated quadratic form reads

$$
(v, v)=\sum_{i=1}^{s} v_{i}^{2}-\sum_{i=s+1}^{n} v_{i}^{2}
$$

Consider the $2^{n}$-dimensional real linear space $C\left(V_{(n, s)}\right)$ with basis

$$
\left\{e_{A}=e_{h_{1}, \cdots, h_{r}}: A=\left(h_{1}, \cdots, h_{r}\right) \in P N, 1 \leq h_{1}<\cdots<h_{r} \leq n\right\}
$$

where $N$ stands for the set $\{1, \cdots, n\}$ and $e_{\emptyset}=e_{0}$.

Now a product on $C\left(V_{(n, s)}\right)$ is defined by

$$
e_{A} e_{B}=(-1)^{n((A \cap B) \backslash S)}(-1)^{p(A, B)} e_{A \triangle B}
$$

where $S$ stands for the set $\{1, \cdots, n\}, n(A)=|A|$,

$$
p(A, B)=\sum_{j \in B} p(A, j), p(A, j)=\{i \in A: i>j\}
$$

and the sets $A, B$ and $A \triangle B$ are ordered in the prescribed way. It is easy to check that $C\left(V_{n, s}\right)$ turned into a linear, associative, but non-commutative algebra over $R$; it is called the universal Clifford algebra over $V_{(n, s)}$. 
In this paper, we use the real Clifford algebra $R_{n, s}$, which means that in $R^{n}$ the basis $\left\{e_{1}, \cdots, e_{n}\right\}$ satisfies $e_{i} e_{i}=1, i=1, \cdots, s$, and $e_{i} e_{i}=-1, i=s+1, \cdots, n$. When $s=0$, we simply set $R_{n, s}=R_{n}$.

If $f$ is Lipschitz continuous, then at any point of differentiability $x \in U$ ( $U$ is an open subset of $R^{n+1}$ ) of $f(x)=\sum_{I} f_{I}(x) e_{I}$, we have

$$
\begin{gathered}
(D f)(x)=\sum_{I} \sum_{j=0}^{n} \frac{\partial f_{I}}{\partial x_{j}}(x) e_{j} e_{I}, \quad(f D)(x)=\sum_{I} \sum_{j=0}^{n} \frac{\partial f_{I}}{\partial x_{j}}(x) e_{I} e_{j}, \\
D^{k} f=D\left(D^{k-1}\right) f, f D^{k}=\left(f D^{k-1}\right) D .
\end{gathered}
$$

We shall call $f$ left $k$-monogenic (right $k$-monogenic, or two-sided $k$-monogenic, respectively) if $D^{k} f=0$ ( $f D^{k}=0$, or $D^{k} f=f D^{k}=0$, respectively); $D$ is called the Cauchy-Riemann operator.

\section{HARDY SPACE $H^{(k)}\left(R^{n}, R_{n, s}\right)$}

Definition 1. Let $R_{n, s}$ be a real Clifford algebra. A Clifford module $L^{2}\left(R^{n}, R_{n, s}\right)$ is defined to be

$$
L^{2}\left(R^{n}, R_{n, s}\right)=\left\{f: R^{n} \rightarrow R_{n, s}, f(x)=\sum_{I} f_{I}(x) e_{I} \mid, f_{I} \in L^{2}\left(R^{n}, R\right), \forall I\right\} .
$$

The Hardy space $H^{(k)}\left(R^{n}, R_{n, s}\right)$ is defined to be

$$
\begin{aligned}
H^{(k)}\left(R^{n}, R_{n, s}\right)=\left\{f \in L^{2}\left(R^{n}, R_{n, s}\right) \mid\right. & F\left(x_{0}, x\right)=P_{x_{0}} * f(x), x_{0}>0, \\
D^{k-1} F\left(x, x_{0}\right) & \left.\neq 0, D^{k} F\left(x_{0}, x\right)=0\right\},
\end{aligned}
$$

where $k \in N, 0 \leq s \leq n$, and $P_{x_{0}}$ is the Poisson kernel.

In these Hilbert spaces, the inner product is denoted by

$$
(f, g)=\int_{R^{n}} \sum_{I} f_{I}(x) g_{I}(x) d x
$$

where, $f(x), g(x) \in L^{2}\left(R^{n}, R_{n, s}\right)$.

In detail, for arbitrary $f(x)=\sum_{I} f_{I}(x) e_{I} \in H^{(1)}\left(R^{n}, R_{n, s}\right)$, let

$$
F\left(x_{0}, x\right)=P_{x_{0}} * f(x)=\sum_{I}\left(P_{x_{0}} * f_{I}(x)\right) e_{I}
$$

and

Then we have

$$
F_{I}\left(x_{0}, x\right)=P_{x_{0}} * f_{I}(x), \partial_{j} F_{I}\left(x, x_{0}\right)=\frac{\partial F_{I}\left(x, x_{0}\right)}{\partial x_{j}} .
$$

$$
F\left(x_{0}, x\right)=\sum_{I} F_{I}\left(x_{0}, x\right) e_{I}, D F\left(x_{0}, x\right)=\sum_{I} \sum_{j=0}^{n} \partial_{j} F_{I} e_{j} e_{I} .
$$

The latter can be rewritten as

$$
D F\left(x_{0}, x\right)=\left(e_{0}, e_{1}, \cdots, e_{n}, \cdots, e_{(1,2, \cdots, n)}\right) A\left(F_{0}, F_{1}, \cdots, F_{n}, \cdots, F_{(1,2, \cdots, n)}\right)^{T},
$$

where $e_{0}, e_{1}, \cdots, e_{n}, \cdots, e_{(1,2, \cdots, n)}$ is an ordered basis of $R_{n, s}$. It is arranged according to $I$ in lexicographical order. $A$ is a $2^{n} \times 2^{n}$ matrix uniquely determined when the order of this basis is set. 
Example 1. $H^{(1)}\left(R^{2}, R_{2,1}\right)=\left\{f \in L^{2}\left(R^{2}, R_{2,1}\right) \mid F\left(x_{0}, x\right)=P_{x_{0}} * f(x), x_{0}>\right.$ $\left.0, D F\left(x_{0}, x\right)=0\right\}, D F=\left(e_{0}, e_{1}, e_{2}, e_{12}\right) A\left(F_{0}, F_{1}, F_{2}, F_{12}\right)^{T}$, where

$$
A=\left[\begin{array}{cccc}
\partial_{0} & \partial_{1} & -\partial_{2} & 0 \\
\partial_{1} & \partial_{0} & 0 & \partial_{2} \\
\partial_{2} & 0 & \partial_{0} & \partial_{1} \\
0 & -\partial_{2} & \partial_{1} & \partial_{0}
\end{array}\right] .
$$

3.1. Characterization of $H^{(k)}\left(R^{n}, R_{n, s}\right)$.

Theorem 1. In the Hardy space $H^{(1)}\left(R^{n}, R_{n}\right)=\left\{f \in L^{2}\left(R^{n}, R_{n}\right) \mid F\left(x_{0}, x\right)=\right.$ $\left.P_{x_{0}} * f(x), x_{0}>0, D^{k-1} F\left(x_{0}, x\right) \neq 0, D F\left(x_{0}, x\right)=0\right\}$, each function $f=\sum_{I} f_{I} e_{I}$ is determined by its $2^{n-1}$ linearly independent function components. Explicitly for $\Omega=\left\{A=\left(h_{1}, \cdots, h_{r}\right) \in P N, 1 \leq h_{1}<\cdots<h_{r} \leq n\right\}$, denote $\Lambda=\{A=$ $\left.\left(h_{1}, h_{2}, \cdots, h_{2 k+1}\right), 1 \leq h_{1}<\cdots<h_{2 k+1} \leq n\right\}$ when $n=2 m, k=0,1, \cdots, m-1$, when $n=2 m+1, k=0,1, \cdots, m$. Then each $f_{J}$ can be represented by the Riesz transforms of all $f_{K} ; f_{K}$ can also be represented by the Riesz transforms of all $f_{J}$, where $J \in \Lambda, K \in \Omega \backslash \Lambda$.

Proof. For convenience, we consider DF in its Fourier transform

$$
\widehat{D F}\left(x_{0}, \xi\right)=\sum_{I} \sum_{j=0}^{n} \widehat{\partial_{j} F_{I}}\left(x_{0}, \xi\right) e_{j} e_{I}
$$

Since $\widehat{\partial_{0} F_{I}}\left(x_{0}, \xi\right)=-|\xi| \widehat{F_{I}}\left(x_{0}, \xi\right), \widehat{\partial_{j} F_{I}}\left(x_{0}, \xi\right)=i \xi_{j} \widehat{F_{I}}\left(x_{0}, \xi\right), j=1,2, \cdots, n$, for all $I$, then

$$
\begin{aligned}
& \widehat{D F}\left(x_{0}, \xi\right)=\sum_{I}\left(-|\xi| \widehat{F_{I}} e_{0}+\sum_{j=1}^{n} i \xi_{j} \widehat{F}_{I} e_{j}\right) e_{I} \\
& =\left(e_{0}, e_{1}, \cdots, e_{I}, \cdots, e_{(1,2, \cdots, n)}\right) B\left(\widehat{F_{0}}, \widehat{F}_{1}, \cdots, \widehat{F}_{I}, \cdots, \widehat{F}_{(1,2, \cdots, n)}\right)^{T} .
\end{aligned}
$$

1) Let us prove that $B$ is a Hermitian matrix. Denote

$$
B=\left(u_{0}, u_{1}, \cdots, u_{I}, \cdots, u_{(1,2, \cdots, n)}\right)^{T}=\left(v_{0}, v_{1}, \cdots, v_{I}, \cdots, v_{(1,2, \cdots, n)}\right),
$$

where $u_{0}, u_{1}, \cdots, u_{I}, \cdots, u_{(1,2, \cdots, n)}$ are the rows of $B$, and $v_{0}, v_{1}, \cdots, v_{I}, \cdots$, $v_{(1,2, \cdots, n)}$ are the columns of $B$.

Let

$$
\overrightarrow{u_{0}}=-|\xi| e_{0}-\sum_{j=1}^{n} i \xi_{j} e_{j}, \overrightarrow{u_{I}}=\overrightarrow{u_{0}} e_{I}, \overrightarrow{w_{0}}=-|\xi| e_{0}+\sum_{j=1}^{n} i \xi_{j} e_{j}, \overrightarrow{w_{I}}=\overrightarrow{w_{0}} e_{I},
$$

for all $I$.

We will prove that $u_{I}$ are the coordinates of $\overrightarrow{u_{I}}$ with respect to the basis $\left\{e_{0}, e_{1}\right.$, $\left.\cdots, e_{(1,2, \cdots, n)}\right\}$, and $v_{I}$ are the transpose of $w_{I}$. Here $w_{I}$ are the coordinates of $\overrightarrow{w_{I}}$ with respect to the basis, $I=(0),(1), \cdots,(1,2, \cdots, n)$.

In fact, let $\lambda=\left(\widehat{F}_{0}, \widehat{F}_{1}, \cdots, \widehat{F}_{I}, \cdots, \widehat{F}_{(1,2, \cdots, n)}\right)^{T}$. From (1), we have

$$
\widehat{D F}=\left(e_{0}, e_{1}, \cdots, e_{(1,2, \cdots, n)}\right)\left(u_{0} \lambda, u_{1} \lambda, \cdots, u_{I} \lambda, \cdots, u_{(1,2, \cdots, n)} \lambda\right)^{T} .
$$

Obviously, $u_{I} \lambda=u_{I}\left(\widehat{F}_{0}, \widehat{F}_{1}, \cdots, \widehat{F}_{I}, \cdots, \widehat{F}_{(1,2, \cdots, n)}\right)^{T}$ is the coefficient of $e_{I}$ in $\widehat{D F}$, for each $I$. 
On the other hand, for each $j=1,2, \cdots, n$, there is a unique $I_{j}$ satisfying $e_{j} e_{I_{j}}=(-1)^{I_{j}} e_{I}$.

From (1), the $e_{I}$ component in $\widehat{D F}$ is

$$
-|\xi| \widehat{F}_{I} e_{I}+\sum_{j=1}^{n} i \xi_{j} \widehat{F}_{I_{j}} e_{j} e_{I_{j}}=\left(-|\xi| \widehat{F}_{I}+\sum_{j=1}^{n}(-1)^{I_{j}} i \xi_{j} \widehat{F}_{I_{j}}\right) e_{I},
$$

so

$$
u_{I} \lambda=-|\xi| \widehat{F}_{I}+\sum_{j=1}^{n}(-1)^{I_{j}} i \xi_{j} \widehat{F}_{I_{j}}
$$

and

$$
\begin{aligned}
& \overrightarrow{u_{I}}=-|\xi| e_{0} e_{I}-\sum_{j=1}^{n} i \xi_{j} e_{j} e_{I} \\
& =-|\xi| e_{0} e_{I}-\sum_{j=1}^{n}(-1)^{I_{j}+1} i \xi_{j} e_{I_{j}}=-|\xi| e_{I}+\sum_{j=1}^{n}(-1)^{I_{j}} i \xi_{j} e_{I_{j}} .
\end{aligned}
$$

From (2) and (3), we obtain that $u_{I}$ is the component of $\overrightarrow{u_{I}}$ with respect to the basis $\forall I$.

Now to prove that $v_{I}$ is the transpose of $w_{I}$, i.e. to prove

$$
\left(e_{0}, e_{1}, \cdots, e_{(1,2, \cdots, n)}\right) v_{I}=\overrightarrow{w_{J}}
$$

Let $\lambda=(0,0, \cdots, \hat{f}, \cdots, 0)^{T}$ be the vector in $R^{2^{n}}$ with all zeros except for an $\hat{f}$ $\left(\hat{f} \in L^{2}\left(R^{n}, R\right)\right)$ in the $I$ th entry. From (11), we have

$$
\left(-|\xi| e_{0} e_{I}+\sum_{j=1}^{n} i \xi_{j} e_{j} e_{I}\right) \hat{f}=\left(e_{0}, e_{1}, \cdots, e_{I}, \cdots, e_{(1,2, \cdots, n)}\right) v_{I} \hat{f} .
$$

This is $\overrightarrow{w_{I}}=\left(e_{0}, e_{1}, \cdots, e_{I}, \cdots, e_{(1,2, \cdots, n)} v_{I}\right.$, so the transpose of $w_{I}$ is precisely $v_{I}$.

These cases and

$$
\begin{aligned}
& \overrightarrow{u_{I}}=-|\xi| e_{0} e_{I}+\sum_{j=1}^{n}(-1)^{I_{j}} i \xi_{j} e_{I_{j}} \\
& \overrightarrow{w_{I}}=-|\xi| e_{1} e_{I}-\sum_{j=1}^{n}(-1)^{I_{j}} i \xi_{j} e_{I_{j}}
\end{aligned}
$$

show that $u_{I}$ is the conjugate transpose of $v_{I}$ for each $I$. So $\bar{B}^{T}=B$, i.e. $B$ is a Hermitian matrix.

2) Let us prove $B B=-2|\xi| B$. By the properties of Fourier transform, we know that the Cauchy-Riemann operator $D$ corresponds to the operator $\hat{D}=-|\xi|+$ $\sum_{j=1}^{n} i \xi_{j} e_{j}$, and the latter corresponds to $B$, so we get

$$
B B=-2|\xi| B \text {. }
$$

3) We are going to prove that $r_{B}=2^{n-1}$. From (5), we get

$$
\left(B+2|\xi| E_{2^{n}}\right) B=-B^{T} B=0,
$$


i.e. $B B^{T}=\overline{B^{T} B}=0$. Therefore, $-2|\xi|$ and 0 are eigenvalues of $B$. Denote by $V_{0}, V_{-2|\xi|}$ the eigenspace of $B$ associated with $0,-2|\xi|$, respectively; $V_{0}^{\prime}$ is the eigenspace of $B^{T}$ associated with 0 . Then

$$
\operatorname{dim} V_{-2|\xi|}=\operatorname{dim} V_{0}^{\prime} \geq r_{B} .
$$

Applying the Plus Nullity Theorem, we get

$$
r_{B}+\operatorname{dim} V_{0}=2^{n}
$$

and

$$
r_{B^{T}}+\operatorname{dim} V_{0}^{\prime}=2^{n}
$$

Threfore

$$
\operatorname{dim} V_{0}=\operatorname{dim} V_{0}^{\prime}=\operatorname{dim} V_{-2|\xi|} .
$$

Since $B$ is a hermitian matrix, $B$ can be diagonalized. If $B$ has other eigenvalues different from $-2|\xi|$ and 0 , let $d$ be the sum of the dimensions of their eigenspaces. Then

$$
\operatorname{dim} V_{-2|\xi|}+\operatorname{dim} V_{0}+d=2^{n} .
$$

Together with (6) and (7), we have

$$
2^{n}=\operatorname{dim} V_{-2|\xi|}+\operatorname{dim} V_{0}+d \geq 2^{n}+d>2^{n}
$$

which is a contradiction. So the eigenvalues of $B$ are $-2|\xi|$ and 0 , and we get

$$
\operatorname{dim} V_{-2|\xi|}=r_{B}
$$

Thus from $\operatorname{dim} V_{-2|\xi|}=\operatorname{dim} V_{0}^{\prime}$ and (7), we know $r_{B}=2^{n-1}$. Therefore each $f$ can be determined by $2^{n-1}$ of its function components.

In fact, the rows $u_{J}$ are the maximum linearly independent in all rows of $B$, where

$$
J \in \Lambda=\left\{A=\left(h_{1}, h_{2}, \cdots, h_{2 k+1}\right), 1 \leq h_{1}<\cdots<h_{2 k+1} \leq n\right\} .
$$

This can be deduced from $\overrightarrow{u_{J}}=-|\xi| e_{J}-\sum_{j=1}^{n} i \xi_{j} e_{j} e_{J}$.

If

$$
\sum_{J \in \Lambda} a_{J} u_{J}=0
$$

there must be an $a_{J}=0$. Hence the $u_{J}$ are linearly independent and, together with $r_{B}=2^{n-1}$, we know all the rows of $B$ can be determined by these $u_{J}$.

On the other hand, for each $j=1, \cdots, n$, there is a $J_{j} \in \Omega \backslash \Lambda$, which uniquely satisfies $e_{j} e_{J}=(-1)^{J_{j}} J_{j}$, so

$$
\overrightarrow{u_{J}}=-|\xi| e_{J}-\sum_{j=1}^{n}(-1)^{J_{j}} i \xi_{j} e_{J_{j}} .
$$

Solving the equations $u_{J}\left(\widehat{F_{0}}, \widehat{F_{1}}, \cdots, \widehat{F_{(1, \cdots, n)}}\right)^{T}=0$, we get

$$
f_{J}(x)=-\sum_{j=1}^{n}(-1)^{J_{j}} R_{j} f_{J_{j}},
$$

where $J \in \Lambda$, i.e. each $f_{J}$ can be represented by the Riesz transforms of all $f_{K}$, where $J \in \Lambda, K \in \Omega \backslash \Lambda$. Similarly, $f_{K}$ can be represented by the Riesz transforms of all $f_{J}$. The proof is finished. 
Remark. From the proof of the above theorem, we can write each function $f \in$ $H^{(1)}\left(R^{n}, R_{n}\right)$ as follows:

1) When $n$ is even,

$$
\begin{aligned}
& f=\sum_{I} f_{I} e_{I} \\
& =\left(f_{0}+R_{1} f_{0} e_{1}+R_{2} f_{0} e_{2}+\cdots+R_{n} f_{n} e_{n}\right) \\
& +\left(f_{12}+R_{1} f_{12} e_{1}+R_{2} f_{12} e_{2}+\cdots+R_{n} f_{12} e_{n}\right) e_{12}+\cdots \\
& +\left(f_{n-1, n}+R_{1} f_{n-1, n} e_{1}+R_{2} f_{n-1, n} e_{2}+\cdots+R_{n} f_{n-1, n} e_{n}\right) e_{n-1, n}+\cdots \\
& +\left(f_{12 \cdots n}+R_{1} f_{12 \cdots n} e_{1}+R_{2} f_{12 \cdots n} e_{2}+\cdots+R_{n} f_{12 \cdots n} e_{n}\right) e_{12 \cdots n} .
\end{aligned}
$$

2) When $n$ is odd,

$$
\begin{aligned}
& f=\sum_{I} f_{I} e_{I} \\
& =\left(f_{0}+R_{1} f_{0} e_{1}+R_{2} f_{0} e_{2}+\cdots+R_{n} f_{n} e_{n}\right) \\
& +\left(f_{12}+R_{1} f_{12} e_{1}+R_{2} f_{12} e_{2}+\cdots+R_{n} f_{12} e_{n}\right) e_{12}+\cdots \\
& +\left(f_{n-1, n}+R_{1} f_{n-1, n} e_{1}+R_{2} f_{n-1, n} e_{2}+\cdots+R_{n} f_{n-1, n} e_{n}\right) e_{n-1, n}+\cdots \\
& +\left(f_{2 \cdots n}+R_{1} f_{2 \cdots n} e_{1}+R_{2} f_{2 \cdots n} e_{2}+\cdots+R_{n} f_{2 \cdots n} e_{n}\right) e_{2 \cdots n} .
\end{aligned}
$$

In fact, we can represent the function by using other function components.

Example 2. The Hardy space $H^{(1)}\left(R^{1}, R_{1}\right)=\left\{f \in L^{2}\left(R^{1}, R_{1}\right) \mid F\left(x_{0}, x\right)=\right.$ $\left.P_{x_{0}} * f(x), x_{0}>0, D F\left(x_{0}, x\right)=0\right\}$ is exactly the classical Hardy space. For each $f \in H^{(1)}\left(R^{1}, R_{1}\right)$, it can be represented by the Hilbert transform (when $n=1$, there is only one Riesz transform, namely the Hilbert transform) of one of its function components.

Example 3. For each $f \in H^{(1)}\left(R^{3}, R_{3}\right)$,

$$
\begin{aligned}
& \widehat{D F}\left(x_{0}, \xi\right) \\
& =\left(e_{0}, e_{1}, e_{2}, e_{3}, e_{(1,2)}, e_{(1,3)}, e_{(2,3)}, e_{(1,2,3)}\right) \\
& B\left(\widehat{F}_{0}, \widehat{F}_{1}, \widehat{F}_{2}, \widehat{F}_{3}, \widehat{F}_{(1,2)}, \widehat{F}_{(1,3)}, \widehat{F}_{(2,3)}, \widehat{F}_{(1,2,3)}\right)^{T},
\end{aligned}
$$

where

$$
B=\left[\begin{array}{c}
u_{0} \\
u_{1} \\
u_{2} \\
u_{3} \\
u_{(1,2)} \\
u_{(1,3)} \\
u_{(2,3)} \\
u_{(1,2,3)}
\end{array}\right]=\left[\begin{array}{cccccccc}
-|\xi| & -i \xi_{1} & -i \xi_{2} & -i \xi_{3} & 0 & 0 & 0 & 0 \\
i \xi_{1} & -|\xi| & 0 & 0 & i \xi_{2} & i \xi_{3} & 0 & 0 \\
i \xi_{2} & 0 & -|\xi| & 0 & -i \xi_{1} & 0 & i \xi_{3} & 0 \\
i \xi_{3} & 0 & 0 & -|\xi| & 0 & -i \xi_{1} & -i \xi_{2} & 0 \\
0 & -i \xi_{2} & i \xi_{1} & 0 & -|\xi| & 0 & 0 & -i \xi_{3} \\
0 & -i \xi_{3} & 0 & i \xi_{1} & 0 & -|\xi| & 0 & i \xi_{2} \\
0 & 0 & -i \xi_{3} & i \xi_{2} & 0 & 0 & -|\xi| & -i \xi_{1} \\
0 & 0 & 0 & 0 & i \xi_{3} & -i \xi_{2} & i \xi_{1} & -|\xi|
\end{array}\right]
$$


By using Theorem 1,

$$
\begin{gathered}
f_{1}(x)=R_{1} f_{0}(x)+R_{2} f_{(1,2)}(x)+R_{3} f_{(1,3)}(x), \\
f_{2}(x)=R_{2} f_{0}(x)-R_{1} f_{(1,2)}(x)+R_{3} f_{(2,3)}(x), \\
f_{3}(x)=R_{3} f_{0}(x)-R_{1} f_{(1,3)}(x)-R_{2} f_{(2,3)}(x), \\
f_{(1,2,3)}(x)=R_{3} f_{(1,2)}(x)-R_{2} f_{(1,3)}(x)+R_{1} f_{(2,3)}(x) .
\end{gathered}
$$

In fact, $f_{0}, f_{(1,2)}, f_{(1,3)}, f_{(2,3)}$ can be represented by the Riesz transform of other function components.

Corollary 1. In the Hardy space $H^{(k)}\left(R^{n}, R_{n}\right)=\left\{f(x) \in L^{2}\left(R^{n}, R_{n}\right) \mid F\left(x_{0}, x\right)=\right.$ $\left.P_{x_{0}} * f(x), x_{0}>0, D^{k-1} F\left(x_{0}, x\right) \neq 0, D^{k} F\left(x_{0}, x\right)=0\right\}$, each $f$ is determined by $2^{n-1}$ linearly independent function components.

Proof. Let $F\left(x_{0}, x\right)=\sum_{I} F_{I}\left(x_{0}, x\right) e_{I}$ and $D F\left(x_{0}, x\right)=\sum_{I} \sum_{j=0}^{n} \partial_{j} F_{I} e_{j} e_{I}$. For convenience, we consider $D F$ in its Fourier transform. Using the formula

$\widehat{D F}\left(x_{0}, \xi\right)=\left(e_{0}, e_{1}, \cdots, e_{I}, \cdots, e_{(1,2, \cdots, n)}\right) B\left(\widehat{F_{0}}, \widehat{F_{1}}, \cdots, \widehat{F_{I}}, \cdots, \widehat{F}_{(1,2, \cdots, n)}\right)^{T}$

$k$ times, we obtain

$$
\widehat{D^{k} F}\left(x_{o}, \xi\right)=\left(e_{0}, e_{1}, \cdots, e_{I}, \cdots, e_{(1,2, \cdots, n)}\right) B^{k}\left(\widehat{F_{0}}, \widehat{F_{1}}, \cdots, \widehat{F_{I}}, \cdots, \widehat{F}_{(1,2, \cdots, n)}\right)^{T} .
$$

Now we prove $r_{B^{k}}=r_{B}=2^{n-1}$. From Theorem 1, we know $B B=-2|\xi| B$. Therefore

$$
B^{k}=-2|\xi| B^{k-1}=(-2|\xi|)^{2} B^{k-2}=\cdots=(-2|\xi|)^{k-1} B .
$$

Then $r_{B^{k}}=2^{n-1}$. This ends the proof.

Theorem 2. $H^{(1)}\left(R^{n}, R_{n, n}\right)=\{0\}$.

Proof. For convenience, we still consider $D F$ in its Fourier transform. Denote $B=$ $\left(u_{0}, u_{1}, \cdots u_{I}, \cdots, u_{(12 \cdots n)}\right)^{T}=\left(v_{0}, v_{1}, \cdots, v_{I}, \cdots, v_{(1,2, \cdots, n)}\right)$. Let $\overrightarrow{u_{0}}=-|\xi| e_{0}+$ $\sum_{j=1}^{n} i \xi_{j} e_{j}, \overrightarrow{u_{I}}=\overrightarrow{u_{0}} e_{I}$, and $\overrightarrow{w_{0}}=-|\xi| e_{0}+\sum_{j=1}^{n} i \xi_{j} e_{j}, \overrightarrow{w_{I}}=\overrightarrow{w_{0}} e_{I}$, for all $I$.

As in Theorem 1, we can prove that $u_{I}$ are the coordinates of $\vec{u}_{I}$ with respect to the basis $\left\{e_{0}, e_{1}, \cdots, e_{(1,2, \cdots, n)}\right\}$, and $v_{I}$ are the transpose of $w_{I}$. Here $w_{I}$ are the coordinates of $\overrightarrow{w_{I}}$ with respect to the basis, $I=(0),(1), \cdots,(1,2, \cdots, n)$. Then we get

1) $B$ is a symmetric matrix.

2) $B \bar{B}=2|\xi|^{2} E_{2^{n}}$.

The method to prove this is similar to Theorem 1.

Therefore $r_{B}=2^{n}$, i.e. $F\left(x_{0}, x\right)=0$. Thus $H^{(1)}\left(R^{n}, R_{n, n}\right)=\{0\}$.

\section{Corollary 2.}

$$
H^{(k)}\left(R^{n}, R_{n, n}\right)=\{0\}, k \in N .
$$

More generally, we have the following theorem.

Theorem 3. For each $f \in H^{(1)}\left(R^{n}, R_{n, s}\right)=\left\{f \in L^{2}\left(R^{n}, R_{n, s}\right) \mid F=P_{x_{0}} * f, x_{0}>\right.$ $\left.0, D F\left(x_{0}, x\right)=0\right\}$, if $\sum_{j=1}^{s} x_{j}^{2} \neq 0$, then $f=0$; otherwise, $f$ can be determined by $2^{n-1}$ linearly independent function components. 
Proof. We consider DF in its Fourier transform. Denote

$$
B=\left(u_{0}, u_{1}, \cdots, u_{I}, \cdots, u_{(12 \cdots n)}\right)^{T}=\left(v_{0}, v_{1}, \cdots, v_{I}, \cdots, v_{(1,2, \cdots, n)}\right) .
$$

Using a method similar to that in Theorem 1 , we get $B \bar{B}=2 \sum_{j=1}^{s} \xi_{j}^{2} E_{2^{n}}$. If $\sum_{j=1}^{s} \xi_{j}^{2} \neq 0$, then $r_{B}=2^{n}$. Therefore $f=0$. If $\sum_{j=1}^{s} \xi_{j}^{2}=0$, then $B$ is a hermitian matrix. Using Theorem 1 , we have $r_{B}=2^{n-1}$. Therefore $f$ is determined by $2^{n-1}$ linearly independent function coefficients.

Corollary 3. For each $f \in H^{(k)}\left(R^{n}, R_{n, s}\right)=\left\{f \in L^{2}\left(R^{n}, R_{n, s}\right) \mid F=P_{x_{0}} * f, x_{0}>\right.$ $\left.0, D^{k-1} F\left(x_{0}, x\right) \neq 0, D^{k} F\left(x_{0}, x\right)=0\right\}$, if $\sum_{j=1}^{s} x_{j}^{2} \neq 0$, then $f=0$; otherwise, $f$ can be determined by $2^{n-1}$ linearly independent function components.

Similarly, we denote the conjugate Hardy space $\bar{H}^{(k)}\left(R^{n}, R_{n, s}\right)$ by

$$
\begin{aligned}
\bar{H}^{(k)}\left(R^{n}, R_{n, s}\right)=\left\{f \in L^{2}\left(R^{n}, R_{n, s}\right) \mid\right. & F\left(x_{0}, x\right)=P_{x_{0}} * f(x), \\
& \left.x_{0}>0, \bar{D}^{k-1} F\left(x_{0}, x\right) \neq 0, \bar{D}^{k} F=0\right\},
\end{aligned}
$$

where $k \in N, 0 \leq s \leq n$.

From the above theorems, we have

Corollary 4. In the conjugate Hardy space $\bar{H}^{(k)}\left(R^{n}, R_{n}\right)=\left\{f(x) \in L^{2}\left(R^{n}, R_{n}\right) \mid\right.$ $\left.F\left(x_{0}, x\right)=P_{x_{0}} * f(x), x_{0}>0, \bar{D}^{k-1} F\left(x_{0}, x\right) \neq 0 \bar{D}^{k} F\left(x_{0}, x\right)=0\right\}$, each $f$ is determined by $2^{n-1}$ linearly independent function components.

Corollary 5. $\bar{H}^{(k)}\left(R^{n}, R_{n, n}\right)=\{0\}$.

F. Sommen proved a decomposition of $L^{2}\left(R^{n}, R_{n}\right)$ first in [12]. The inner product he used was Clifford algebra-valued. Here we can get the orthogonal decomposition of $L^{2}\left(R^{n}, R_{n}\right)$ by using our characterization of Hardy space and conjugate Hardy space:

$$
L^{2}\left(R^{n}, R_{n}\right)=H^{(1)}\left(R^{n}, R_{n}\right) \oplus \bar{H}^{(1)}\left(R^{n}, R_{n}\right) .
$$

Now we give a natural method to obtain some compensated quantities. For each $f, g \in H^{(1)}\left(R^{n}, R_{n}\right)$, for convenience, we assume $n$ is an even number. By using (8), in the product $f g$, we have

$$
\begin{aligned}
& R f_{0} R g_{I} e_{I} \\
= & \left(f_{0}+R_{1} f_{0} e_{1}+R_{2} f_{0} e_{2}+\cdots+R_{n} f_{0} e_{n}\right) \\
& \cdot\left(g_{I}+R_{1} g_{I} e_{1}+R_{2} g_{I} e_{2}+\cdots+R_{n} g_{I} e_{n}\right) e_{I} \\
= & {\left[\left(f_{0} g_{I}-\sum_{j=1}^{n} R_{j} f_{0} R_{j} g_{I}\right)+\cdots+\sum_{j, k=1, j<k}^{n}\left(R_{j} f_{0} R_{k} g_{I}-R_{k} f_{0} R_{j} g_{I}\right) e_{j k}\right] e_{I} }
\end{aligned}
$$


and

$$
\begin{aligned}
& R f_{I} e_{I} R g_{J} e_{J} \\
= & \left(f_{I}+R_{1} f_{I} e_{1}+R_{2} f_{I} e_{2}+\cdots+R_{n} f_{I} e_{n}\right) e_{I} \\
& \cdot\left(g_{J}+R_{1} g_{J} e_{1}+R_{2} g_{J} e_{2}+\cdots+R_{n} g_{J} e_{n}\right) e_{J} \\
= & \left(f_{I}+R_{1} f_{I} e_{1}+R_{2} f_{I} e_{2}+\cdots+R_{n} f_{I} e_{n}\right) \\
& \cdot\left(g_{J}-\sum_{j \in I} R_{j} g_{J} e_{j}+\sum_{j \notin I} R_{j} g_{J} e_{j}\right) e_{I} e_{J} \\
= & {\left[\left(f_{I} g_{J}+\sum_{j \in I} R_{j} f_{I} R_{j} g_{J}-\sum_{j \notin I} R_{j} f_{I} R_{j} g_{J}\right)\right.} \\
& +\sum_{j \in I}\left(g_{J} R_{j} f_{I}-f_{I} R_{j} g_{J}\right) e_{j} \\
& +\sum_{k \notin I}\left(g_{J} R_{k} f_{I}+f_{I} R_{k} g_{J}\right) e_{k} \\
& +\sum_{j \in I, k \notin I, j<k}\left(R_{j} f_{I} R_{k} g_{J}+R_{k} f_{I} R_{j} g_{J}\right) e_{j k} \\
& +\sum_{k, j \notin I, j<k}\left(R_{j} f_{I} R_{k} g_{J}-R_{k} f_{I} R_{j} g_{J}\right) e_{j k} \\
& \left.-\sum_{j, k \in I, j<k}\left(R_{j} f_{I} R_{k} g_{J}-R_{k} f_{I} R_{j} g_{J}\right) e_{j k}\right] e_{I} e_{J},
\end{aligned}
$$

where $I \in \Omega \backslash \Lambda, I \neq\{0\}$.

If we denote

$$
\begin{aligned}
& Q_{0}(u, v)=u v-\sum_{j=1}^{n} R_{j} u R_{j} v, Q_{j}(u, v)=u R_{j} v-R_{j} u v, \\
& Q_{j, k}(u, v)=R_{j} u R_{k} v-R_{k} u R_{j} v, \\
& P_{0}(u, v)=u v+\sum_{j \in I} R_{j} u R_{j} v-\sum_{k \notin I} R_{k} u R_{k} v, P_{j}(u, v)=R_{j} u v-u R_{1} v, \\
& P_{j, k}(u, v)=R_{j} u R_{k} v+R_{k} u R_{j} v,
\end{aligned}
$$

where $j, k=1, \cdots, n, j<k$, for real-valued functions $u, v \in L^{2}\left(R^{n}\right)$, then in the product $f g$, there are six kinds of components as above.

For a real-valued symbol function $b$, write

$$
\begin{gathered}
\left\langle Q_{j}(u, v), b\right\rangle=\left\langle H_{b}^{j} u, v\right\rangle,\left\langle P_{j}(u, v), b\right\rangle=\left\langle T_{b}^{j} u, g\right\rangle, \text { where } j=0,1, \cdots, n, \\
\left\langle Q_{j, k}(u, v), b\right\rangle=\left\langle H_{b}^{j, k} u, v\right\rangle,\left\langle P_{j, k}(u, v), b\right\rangle=\left\langle T_{b}^{j, k} u, v\right\rangle,
\end{gathered}
$$

where, $j, k=, 1,2, \cdots, n, j<k$.

Denote the corresponding Fourier kernels of $H_{b}^{j}$ by $A_{j}(\xi, \eta)$, and the Fourier kernels of $H_{b}^{j, k}$ by $A_{j, k}(\xi, \eta)$. Then we obtain

$$
A_{0}(\xi, \eta)=1-\frac{\xi \cdot \eta}{|\xi||\eta|}=\frac{1}{2}\left(\frac{\xi}{|\xi|}-\frac{\eta}{|\eta|}\right)^{2}, A_{j}(\xi, \eta)=-i\left(\frac{\xi_{j}}{|\xi|}-\frac{\eta_{j}}{|\eta|}\right)
$$


where $j=1, \cdots, n$,

$$
A_{j, k}(\xi, \eta)=\frac{\eta_{j} \xi_{k}-\eta_{k} \xi_{j}}{|\xi||\eta|}, j, k=1, \cdots, n, j<k .
$$

Similarly, denote the corresponding Fourier kernels of $T_{b}^{j}, T_{b}^{j, k}$ by $B_{j}(\xi, \eta), j=$ $0,1, \cdots, n$, and $B_{j, k}(\xi, \eta)$, respectively. Then

$$
\begin{gathered}
B_{0}(\xi, \eta)=1+\sum_{j \in I} \frac{\xi_{j} \eta_{j}}{|\xi||\eta|}-\sum_{k \notin I} \frac{\xi_{k} \eta_{k}}{|\xi||\eta|}, B_{j}(\xi, \eta)=i\left(\frac{\xi_{j}}{|\xi|}+\frac{\eta_{j}}{|\eta|}\right), j=1, \cdots, n, \\
B_{j, k}(\xi, \eta)=\frac{\eta_{j} \xi_{k}+\eta_{k} \xi_{j}}{|\xi||\eta|}, j, k=1, \cdots, n, j<k .
\end{gathered}
$$

Therefore we have six kinds of kernels: $A_{0} ; A_{j}, j=1, \cdots, n ; A_{j, k}, j, k=1, \cdots, n$, $j<k ; B_{0} ; B_{j}, j=1, \cdots, n$; and $B_{j, k}, j, k=1, \cdots, n, j<k$.

Using theorems in [8], we get $\left\langle Q_{0}(u, v), b\right\rangle$ is a compensated quantity which belongs to the real Hardy space $H^{1}\left(R^{2}\right)$, and its corresponding paracommutator is bounded if and only if $b \in B M O\left(R^{2}\right)$. It is compact if and only if $b \in V M O\left(R^{2}\right)$; for $\frac{2}{2}=1<p<\infty$, it belongs to $S_{p}$ if and only if $b \in B_{p}\left(R^{2}\right)$; for $0<p \leq \frac{2}{2}=1$, it belongs to $S_{p}$ if and only if $b$ is a polynomial.

$\left\langle Q_{j}(u, v), b\right\rangle, j=1, \cdots, n$, are compensated quantities which belong to the real Hardy space $H^{1}\left(R^{2}\right)$, and their corresponding paracommutators are bounded if and only if $b \in B M O\left(R^{2}\right)$. They are compact if and only if $b \in V M O\left(R^{2}\right)$; for $2<p<\infty$, they belong to $S_{p}$ if and only if $b \in B_{p}\left(R^{2}\right)$; for $0<p \leq 2$, they belong to $S_{p}$ if and only if $b$ is a polynomial.

Similarly $Q_{j, k}, j, k=1, \cdots, n, j<k$, have the same properties. The quantities $P_{j}(u, v), j=0,1, \cdots, n, P_{j, k}(u, v), j, k=1, \cdots, n j<k$, are not compensated, they belong to $L^{1}\left(R^{2}\right)$ only. Their corresponding paracommutators are bounded iff $b \in L^{\infty}\left(R^{2}\right)$, and can never be compact unless $b \equiv 0$.

\section{REFERENCES}

1. S. Bernstein, Operator Calculus for Elliptic Boundary Value Problems in Unbounded Domains, Zeitschrift für analysis und ihre Anwendungen, 10, (1991), 447-460. MR 93e:35015

2. F. Brackx, R. Delanghe, and F. Sommen, Clifford Analysis, Pitman Advanced Publishing Program, Boston, MA, 1982. MR 85j:30103

3. R. Delanghe and F. Brackx, Hypercomplex function theory and Hilbert modules with reproducing kernel, Proc. London Math. Soc.(3) 37, (1978), 545-576. MR 81j:46032

4. K. Gürlebeck and W. Sprößig, Quaternionic Analysis and Elliptic Boundary Value Problems, Birkhäuser-Verlag, Basel, Berlin, 1990. MR 91k:35002b

5. M. Mitrea, Clifford wavelets, singular integrals and Hardy space, Springer-Verlag, Berlin, Heidelberg, 1994. MR 96e:31005

6. S. Janson and J. Peetre, Paracommutators-boundedness and Schatten-von Neumann properties, Trans. Amer. Math. Soc. 305, (1988), 467-504. MR 89g:47034

7. L. Z. Peng, On the compactness of paracommutators, Ark. Mat. 26, (1988), 315-325. MR 91g:47020

8. L. Z. Peng and M. W. Wong, Compensated compactness and paracommutators, J. London Math. Soc., 62:2, (2000), 505-520. MR 2001g:42035

9. T. Qian and J. Ryan, Conformal transformations and Hardy spaces arising in Clifford analysis, J. Operator Theory, 35, (1996), 349-372. MR 97d:30064

10. F. Sommen, A product and an exponential function in hypercomplex function theory, Appl. Anal. 12, (1981), 13-26. MR 84a:30087

11. F. Sommen, Microfunctions with values in a Clifford algebra, 2, Scientific Papers in the College of Arts and Sciences, University of Tokyo, 36, (1986), 15-37. MR 88f:30083 
12. F. Sommen, Hypercomplex Fourier and Laplace Transforms 2, Complex Variables Theory Appl., 1, (1983), 209-238. MR 84g:46061

13. Z. J. Wu, Clifford algebras, Hardy spaces and compensated compactness, Clifford algebras in analysis and related topics (John Ryan, ed.), Studies in Adv. Math., CRC Press, Boca Raton, FL, 1996, 217-238. MR 96m:30067

14. Z. J. Wu, Commutators and related operators on Harmonic Bergman Space of $R_{+}^{n+1}$, J. Funct. Anal. 144, (1997), 448-474. MR 98a:47029

Lmam, School of Mathematical Sciences, Peking University, Beijing, 100871, PeoPLE'S REPUBLIC OF CHINA

E-mail address: lzpeng@pku.edu.cn

Department of Mathematics, Beijing Normal University, Beijing, 100875, People's Republic of China - and - Academy of Mathematics and System Sciences, Chinese Academy of Sciences, 100080, People's Republic of China

E-mail address: jmz70@sina.com 